\title{
Las Aulas virtuales como herramientas facilitadoras de aprendizajes durante el confinamiento por la covid-19 UDELAS
}

\author{
Virtual classrooms as tools learning facilitators during confinement by covid-19 UDELAS
}

\author{
Salas de aula virtuais como ferramentas facilitadores de aprendizagem durante o \\ confinamento por covid-19 UDELAS
}

ARTICULO DE INVESTIGACIÓN

José Guilbauth

losguilbauth_1@hotmail.com

ORCID: 0000-0002-4300-0268

\author{
Itzel P. de Guilbauth \\ veroka2003@hotmail.com \\ ORCID: 0000-0002-2884-5588
}

\section{Universidad Especializada de las Américas, Panamá}

Recibido 01 de octubre 2020 | Arbitrado y aceptado 30 de octubre 2020 | Publicado en diciembre 2020

\section{RESUMEN}

El objetivo de la investigación fue identificar el tipo de herramientas tecnológicas, formas de comunicación y desarrollo de las aulas virtuales empleadas en clases de las Licenciaturas en Estimulación Temprana y Orientación Familiar, Licenciatura en Educación Especial, Licenciatura de Docencia en Inglés y Licenciatura en Informática Educativa en la Facultad de Educación Especial y Pedagogía de la Universidad Especializada de las Américas, Panamá, durante el primer semestre del Año Académico 2020. En base al método descriptivo y con apoyo de la técnica de encuesta se identificaron competencias, actividades y herramientas que poseían docentes y estudiantes al enfrentarse a la formación universitaria virtual. Los resultados determinaron que producto de una decisión repentina en el cambio de modalidad de enseñanza presencial a virtual, producto del COVID 19, se presentaron respuestas no planificadas, pero permisibles a que el acto didáctico se produjera sin contratiempos y de forma continua en la formación universitaria.

Palabras clave: Formación Superior; educación a distancia; herramientas tecnológicas; aulas virtuales, asignaturas

\section{ABSTRACT}

The objective of the research was to identify the type of technological tools, forms of communication and development of virtual classrooms used in classes of the Bachelor's Degree in Early Stimulation and Family Orientation, the Bachelor's Degree in Special Education, the Bachelor's Degree in Teaching in English and the Bachelor's Degree in Educational Informatics in the Faculty of Special Education and Pedagogy of the Specialized University of the Americas, Panama, during the first semester of the Academic Year 2020. Based on the descriptive method and with the support of the survey technique, competencies, activities and tools possessed by teachers were identified and students when facing virtual university training. The results determined that as a result of a sudden decision in the change from face-to-face to virtual teaching modality, a product of COVID 19, unplanned responses were presented, but permissible for the didactic act to occur smoothly and continuously in university education.

Key words: Higher Education; long distance education; Technological tools; virtual classrooms, subjects 


\section{RESUMO}

O objetivo da pesquisa foi identificar o tipo de ferramentas tecnológicas, formas de comunicação e desenvolvimento de salas de aula virtuais utilizadas nas aulas do Bacharelado em Estimulação Precoce e Orientação Familiar, do Bacharelado em Educação Especial, do Bacharelado em Docência da Língua Inglesa e do Bacharelado em Informática Educacional na Faculdade de Educação Especial e Pedagogia da Universidade Especializada das Américas, Panamá, durante o primeiro semestre do Ano Acadêmico de 2020. Com base no método descritivo e com o apoio da técnica de pesquisa, foram identificadas as competências, atividades e ferramentas possuídas pelos professores e alunos quando enfrentam o treinamento em universidade virtual. Os resultados determinaram que, em decorrência de uma decisão repentina de mudança da modalidade de ensino presencial para a virtual, produto do COVID 19, foram apresentadas respostas não planejadas, mas permitidas para que o ato didático ocorresse de maneira suave e contínua no ensino universitário.

Palavras-chave: Ensino Superior; Educação a Distância; Ferramentas tecnológicas; salas de aula virtuais, assuntos

\section{INTRODUCCIÓN}

Una vez que se dieron los primeros descubrimientos en la microelectrónica e innovaciones en la informática, empezaron a producirse los primeros equipos y aplicaciones basadas en la imagen, la digitalización, la velocidad de transmisión, la interactividad y sobre todo la virtualidad. Estas características fueron propias de la denominadas Tecnologías de la Información y Comunicación, las cuales impactaron en gran medida todas las actividades de la denominada Sociedad del Conocimiento; produciéndose una gran necesidad de profesionales y el reto de formar acorde a las competencias de las virtudes y beneficios que ofrecen las Tecnologías de la Información y Comunicación. Al respecto, Díaz Barriga, Rigo y Hernández (2015) expresan que las Tecnologías de la Información y
Comunicación, "son herramientas técnicas o artefactos físicos cuyo empleo facilita o hace más eficientes las formas de acción que ocurrían de otra manera antes de su advenimiento". Las Tecnologías de la Información y Comunicación ha sido un factor clave para el desarrollo del ser humanos y de la sociedad. De allí que la formación de profesionales con competencias y excelentes desempeños en problemáticas sociolaborales ha llevado a los centros de formación universitaria a estructurar sus ofertas basadas en la creación de entornos y comunidades que aprenden con apoyo de las tecnologías de la Información y Comunicación.

Según Díaz Barriga et al. (2015) las Tics, "tiene un sentido de herramientas semiótica o psicológica. Este concepto se produce basado en la Teoría del Aprendizaje Social propuesta por Vygotsky, en donde existe una función mediadora por parte de herramientas. Así una herramienta es semiótica o psicológica, cuando su selección y uso depende de un proceso de análisis y razonamiento, basado en el tipo de contenido a enseñarse, características del usuario y las especificaciones técnicas del funcionamiento de esta.

Otro punto de vista en cuanto al concepto de Tecnología de la Información y Comunicación es señado por Ochoa y Cordero (2002) al expresar que son "un conjunto de procesos y productos derivados de las nuevas herramientas (hardware y software), soportes y canales de comunicación, relacionados con el almacenamiento, procesamiento y la transmisión digitalizada de la información". Sin duda, la definición anterior se enmarca en una teoría de sistemas en donde basados en soportes y canales se puede procesar una información, o sea recibir, analizar transformar, guardar y recuperar para la posible resolución a distintos problemas de la sociedad. En ese mismo orden de ideas encontramos que Thompson y Strickland, (2004) se refiere a las 
tecnologías de información y comunicación, a "aquellos dispositivos, herramientas, equipos y componentes electrónicos, capaces de manipular información que soportan el desarrollo y crecimiento económico de cualquier organización".

Dicho planteamiento está cercano al de Ochoa y Cordero (2002), pues, señalan que son herramienta y equipos que procesan una determinada información para luego ser empleada en el desarrollo de una nación. No lo hace tampoco diferente, la UNAM, la cual define a las Tecnologías de la Información y Comunicación como las que "contemplan al conjunto de herramientas relacionadas con la transmisión, procesamiento y almacenamiento digitalizado de la información, como al conjunto de procesos y productos derivados de las nuevas herramientas (hardware y software), en su utilización en la enseñanza." En términos similares se pronuncia Luna. N. en el Foro Mundial de Economía (2019), Agenda de Weforum, al señalar que "son el conjunto de herramientas relacionadas con la transmisión, procesamiento y almacenamiento digitalizado de la información. Un aliado del emprendimiento, tanto en nuevos conceptos como en lo tradicional". No cabe duda de que las Tecnologías de la Información y Comunicación son herramienta que dentro de un proceso sistémica emiten, procesan, transformar, registran y recuperar información, de allí el gran valor de sus características y que han potencializado su papel dentro de la sociedad. Cabero (2006), al respecto, describe las características que hacen particular la existencia y funcionamiento de last Tecnologías de la Información y Comunicación, las mismas son: Inmaterialidad; Penetración en todos los sectores (culturales, económicos, educativos, industria les, etc.), Interconexión, Interactividad, Instantaneidad, Creación de nuevos lenguajes expresivos, Ruptura de la linealidad expresiva, Elevados parámetros de calidad de imagen y sonido, Potenciación audiencia segmentaria y diferenciada, Digitalización, Más influencia sobre los procesos que sobre los productos, Tendencia hacia la automatización, Diversidad e Innovación.

Estas características blindan a cualquier medio, recurso o tecnología enmarcados dentro de los definidos como tecnologías de la información y comunicación, pues han fortalecido la capacidad de penetración en toda la sociedad, así como la posibilidad de interconexión e interactividad que da una gran fortaleza y capacidad de impactar en cualquier escenario de la sociedad actual. Unida a estas características tenemos la velocidad con la que se conduce la información con una gama variada de signos, símbolos, una calidad en el audio, en la imagen y el sonido producto de la digitalización; convirtiéndose en medios sólidos, versátiles y con una posibilidad de desarrollar actividades que influyen en los procesos de una información en todas las clases y tipos de mercado. Si las tecnologías de la información y comunicación (TIC) actuales son comparadas con las existentes antes de los años 90, puede señalarse en qué consistió su transformación y sitial en cada función del quehacer de la sociedad actual. Sólo la radio en las tecnologías tradicionales logró una penetración y utilidad en los diversos grupos sociales produciendo resultados de gran admiración y replicación. Al respecto, Almenara, y Cejudo (2015) señala que, " ésta fuerte penetración no es la diversidad de herramientas con que cuentan los profesores y los alumnos, las cuales les permiten trabajar con imágenes, sonidos o multimedia, sino la diversidad de escenarios de comunicación que han creado para la enseñanza, que permiten crear nuevos escenarios de formación que vienen caracterizados por ubicar a los estudiantes en el centro del 
escenario formativo, sacar el aprendizaje de concepción aislada e individual y dirigido hacia posiciones sociales y colaborativas".

Todo ese andamiaje de características que poseen las tecnologías de la información y comunicación ha creado nuevas competencias para el desarrollo de actividades en la sociedad obtenidas con otros procedimientos $\mathrm{y}$ metodologías. De allí el gran reto de las instituciones de educación superior de llenar ese vacío que han creado las tecnologías de la información y comunicación en el desempeño laboral de los futuros profesionales y que acuden a los centros en búsqueda de la formación sólida e integral, que produzca futuros profesionales con excelente desempeño en las tareas y puestos de trabajo de la sociedad actual. Ante la responsabilidad de acoger el reto que han creado las Tecnologías de la Información en la sociedad actual, (Aviram, 2002 en Pere Marqués (2013) identifica tres posibles reacciones de los centros docentes para adaptarse a las TIC y al nuevo contexto cultural, señala tres escenarios bastantes definidos a saber, el primer escenario con características tecnócratas en donde se producen pequeños ajuste pata la adopción de la Tics; un segundo escenario que denomina reformista y caracterizado por emplear las Tics en tres dimensiones, aprender sobre las Tics, aprender de las Tics y aprender con las Tics. Estos cambios van acompañados de determinadas funciones de la Tecnologías de la Información y Comunicación. Al respecto, Pere Marqués (2013) expresa que las tecnologías de la Información y comunicación cumplen las siguientes funciones en los centros educativos: Alfabetización digital de los estudiantes (y profesores... y familias...); Uso personal (profesores, alumnos...): acceso a la información, comunicación, gestión y proceso de datos...; Gestión del centro: secretaría, biblioteca, gestión de la tutoría de alumnos...;
Uso didáctico para facilitar los procesos de enseñanza y aprendizaje; Comunicación con las familias (a través de la web de centro...); Comunicación con el entorno; Relación entre profesores de diversos centros (a través de redes y comunidades virtuales): compartir recursos y experiencias, pasar informaciones, preguntas.

El poder manejar adecuadamente las características y funciones técnicas de un abanico de tecnologías en la educación nos empodera de su aplicación y por consiguiente la obtención de resultados de aprendizajes de calidad. Las funciones bien acogidas $\mathrm{y}$ planificadas en la mejora del aprendizaje de estudiantes de un centro educativo, producirá una serie de beneficios, algunos de ellos son mencionados por (Sancho 2006, citado por Diaz Barriga 2001) a saber: a. Su potencial para alterar la estructura de intereses, prioridades y relaciones de poder entre los individuos; b. El cambio que producen en el carácter de los símbolos con los que pensamos; esto da la pauta a hablar de la existencia de una mente virtual (concepto desarrollado en sus implicaciones a la educación por Badia, y Monereo, 2004 y por Hernández, 2019) inimaginable sin la mediación de dicha tecnología); c. Las importantes modificaciones que han introducido en la naturaleza de las comunidades humanas; existencia de ciberespacio, y globalidad, ya sea en condiciones de inclusión o de exclusión de dicha tecnología".

El conocer una tecnología de la información y comunicación, basado en sus funciones y beneficios trae consigo respetar varios elementos que debemos considerar al realizar su selección, entre ellos podemos mencionar, la necesidad de aprendizaje que posee el estudiante. Condición que debe tomarse en cuenta además para seleccionar los contenidos curriculares que se le deben proveer en la formación. El otro elemento de 
gran relevancia es conocer el tipo de aprendizaje que más emplean los estudiantes en el proceso formativo, pues de este dependerá en gran parte el identificar y seleccionar las metodologías que se acoplan a dicho aprendizajes en determinados contenidos. Teniendo este panorama, toca ahora elegir la gama de tecnologías de la información y comunicación al alcance y determinar cuál o cuáles serán las que apoyarán la mejor transmisión, procesamiento, archivo $\mathrm{y}$ recuperación de conocimientos para una posible resolución de problemas.

A propósito, Cabero (2015) señala una serie de criterios para la selección de los medios y expresa que "superan el simple hecho de sus características técnicas y estéticas, como se ha señalado; veamos los mismos: a). Su selección debe hacerse teniendo en cuenta los objetivos y contenidos que se desean alcanzar y transmitir; b). Las predisposiciones que el alumnado $y$ el profesorado tengan hacia ellos pueden condicionar los resultados que se obtengan, y en consecuencia debe ser uno de los criterios que se han de movilizar para su puesta en acción; c). Contemplar las características de los receptores: edad, nivel sociocultural y educativo; d).El contexto instruccional y físico es un elemento condicionador facilitando o dificultándose su inserción; e). Las diferencias cognitivas entre los estudiantes pueden condicionar los resultados que se pretenden alcanzar y las formas de utilización; f). Las TIC deben propiciar la intervención sobre ellas; g). Sus características técnicas y sus parámetros de cualidades son una dimensión por considerar; h). En la medida de lo posible, seleccionar TIC que permitan la participación del profesorado y el alumnado en la construcción de los mensajes. i). Analizar los mensajes contemplando no solo su capacidad como canal, sino también las características de los mensajes que transmite, y sobre todo contemplando los valores transferidos; j). Sus calidades técnicas, facilidad y versatilidad deben ser también contempladas; k). Seleccionar TIC de fácil utilización, l). Y en la medida de lo posible, seleccionar TIC que puedan relacionarse y combinarse con otras".

En síntesis, el clasificar una TIC, conlleva a una decisión en donde el aspecto central para esa toma de decisión es lo que se enseñará, las cualidades del grupo, el alcance y adecuado manejo de estas y su adquisición de forma fácil, de tal manera que ese proceso educativo sea exitoso para toda la comunidad educativa.

Según la UNESCO, en su documento, Educación Superior Digital (s.f.) expresa que "durante los últimos años la enseñanza superior ha experimentado una transformación radical, fundamentalmente en cuanto al auge de la internalización de la movilidad de los estudiantes, la ampliación de las oportunidades de aprendizaje en línea y servicios de internet en todos los países". En el mismo documento citado, la UNESCO, recomienda en el Marco de Acción, "que los gobiernos formulen políticas y programas para brindar una educación superior a distancia de calidad, adecuadamente financiada, basadas en las tecnologías, en particular mediante cursos en línea masivos y de libre acceso que responden a las normas de calidad a fin de mejorar la accesibilidad a estos".

La formación superior basada en las tecnologías de la información y comunicación debe ser uno de los aspectos a considerar en el plan de desarrollo de toda universidad, de allí el avance de la formación superior virtual en los últimos años y ahora el centro generador del aprendizaje durante el confinamiento y suspensión de clases producto de la pandemia mundial por el COVID-19. 
Salinas (citado en Cabero, 2015) define entorno virtual de aprendizaje como "aquél espacio o comunidad organizada con el propósito de lograr el aprendizaje, y que este lugar requiera ciertos componentes: una función pedagógica (que hace referencia a actividades de aprendizaje, a situaciones de enseñanza, a materiales de aprendizaje, al apoyo y tutoría puestos en juego, a la evaluación, etc....), la tecnología apropiada a la misma (que hace referencia a las herramientas seleccionadas en conexión con el modelo pedagógico) y los aspectos organizativos ( que incluye la organización del espacio, del calendario, la gestión de la comunidad, etc.)".

La terminología educación virtual, según Cabero et al. (2015) "se refiere a varias modalidades de comunicación digital y la misma depende del tipo de soporte en que se basa el acto didáctico". De aquí resultan una serie de herramientas virtuales actuales empleadas en la educación superior, basadas en la virtualidad, interactividad y la digitalización, de allí la trascendencia y eficiencia de su utilidad y resultados en la sociedad de hoy. A propósito, la serie de herramientas tecnológicas educativas que debe manejar el docente en la formación virtual, deben ser: El aprendizaje en línea; Los entornos colaborativos; Los medios sociales; El aprendizaje con soporte del celular; La realidad aumentada; El aprendizaje personalizado; El internet de las cosas; Los laboratorios virtuales y remotos.

Todas estas herramientas innovadoras basadas en el funcionamiento de las tecnologías de la información y comunicación exigen del docente una nueva preparación para responder a las necesidades de los estudiantes, los cuales en la mayoría son nativos digitales. Igualmente, no debe perderse de vista que el medio laboral cada vez será más exigente en cuanto al dominio de competencias tanto humanas como digitales, esto se debe gracias a las ventajas de la formación virtual y de las aulas virtuales. Al respecto, Cabero, y otros (2014), señala las siguientes ventajas: se adapta con facilidad a las características y necesidades de los estudiantes, conecta a estudiantes ubicados en lugares geográficos diferentes, el ritmo del aprendizaje es marcado por los estudiantes, es fácil la actualización de los contenidos, ofrece flexibilidad espacio-temporal para la formación; facilita la interactividad del docente con sus estudiantes, de los estudiantes entre sí y de los estudiantes con los objetos de aprendizaje, pueden utilizarse recursos en diferentes formatos: texto, clip de vídeos, podcast de audio, simuladores, pueden utilizarse diferentes tipos de herramientas de comunicación sincrónica y asincrónica para facilitar la interacción entre los diferentes participantes en la acción formativa, se amplían los escenarios formativos, y deslocalización del conocimiento.

Belloch (2012) de igual manera señala que en la educación se ha producido una serie de ventajas al emplear las Tecnologías de la Información y Comunicación, son ellas: Información variada. - Es posible acceder a gran cantidad de información sobre diferentes ámbitos. Esto permite que el alumno deba realizar un análisis de esta que le permitan valorar la calidad y credibilidad de la misma. Flexibilidad instruccional. - El ritmo de aprendizaje y el camino a seguir durante el proceso puede ser diferente para los distintos alumnos adecuándose a las necesidades diversas que se presentan en el aula. Complementariedad de códigos. - Las aplicaciones multimedia, que utilizan diversos códigos de comunicación, permiten que estudiantes con distintas capacidades $\mathrm{y}$ habilidades cognitivas puedan extraer un 
mejor provecho de los aprendizajes realizados. Aumento de la motivación. Diversos estudios muestran que los estudiantes se muestran más motivados cuando utilizan las TIC, este efecto que aún se produce, puede ser efecto de la novedad, aunque personalmente opino que el aumento de la motivación está muy relacionado, tanto con el mayor atractivo de las presentaciones multimedia sobre las tradicionales, como por la mayor implicación del alumno en su proceso de aprendizaje. Actividades colaborativas. - El uso adecuado de las TIC, en trabajos de grupo, puede potenciar las actividades colaborativas y cooperativas entre los alumnos y también la colaboración con otros centros o instituciones por medio de la red. Potenciar la innovación educativa. - La nueva sociedad utiliza nuevas tecnologías que favorecen nuevas metodologías. Si bien no es una relación causa-efecto, es indudable que los profesores que conocen nuevas tecnologías tienden a buscar nuevas formas de enseñar y nuevas metodologías didácticas más adecuadas a la sociedad actual y a los conocimientos y destrezas que deben desarrollar los estudiantes para su adaptación al mundo adulto.

Sin duda que todo proyecto y programa educativo basado en las tecnologías de la información y comunicación, al poseer una serie de características técnicas, ventajas y desventajas, tendrán que valorarse para ser empleadas en determinados casos según la población, contenidos y contextos universitarios. Algunas de esas desventajas según Meneses, (2007) son: la pasividad con la que se pueda afrontar este método de estudio, ya que se puede percibir como algo "más fácil"; Inexistencia de una estructura pedagógica completa con procesos cognitivos y técnicas de aprendizaje; crea una brecha en la relación alumno-maestro, rompiendo los lazos humanos que se puedan generar; Para personas que aprecien el contacto personal y que se vean beneficiados de las relaciones afectivas puede resultar desconcertante; La disciplina y constancia que se requieren en la formación a distancia es mayor; Una mala conexión de internet, falencias en el equipo o material de trabajo pueden generar retrasos e interrupciones, Sin una rutina de estudios programada, puedes descontrolar tus horarios para otras actividades.

Elegir la opción de formación superior bajo la modalidad virtual, requiere una serie de decisiones, las cuales están basadas en los requisitos, perfiles exigidos, condiciones y dinámica a la cual debe someterse el estudiante y el docente. Esto hace necesario que la puesta en marcha de un programa formativo universitario, basado en las tecnologías de la información y comunicación, requiera de mucha planificación, tecnologías, estrategias y una organización, en general que garantice el éxito esperado. $\mathrm{Al}$ respecto Cabero y Barroso, (2015) señalan que para emplear TIC en el proceso de enseñanza y aprendizaje hay que considerar una serie de componentes, los mismos son: "contar con un soporte físico (el hardware), unos contenidos $\mathrm{y}$ mensajes transferidos por las TIC, un propósito educativo que el profesor persigue con su utilización y una estructura organizativa del centro o de la institución educativa donde el medio es insertado". Lo señalado por Cabero y Barroso (2015) lleva a la reflexión que la implementación de un entorno virtual de aprendizaje requiere de planificación, presupuesto, capacitación de todo el engranaje universitario, evaluación permanente y sobre todo de herramientas para responder a las exigencias de la población estudiantil y el profesorado. 
Boneu (2007) citado por Belloch (2013) señala cuatro características básicas que debe poseer toda plataforma e-learning, las mismas son: Interactividad. Conseguir que la persona que está usando la plataforma tenga conciencia de que es el protagonista de su formación. Flexibilidad. Conjunto de funcionalidades que permite que el sistema de e- learning tenga una adaptación fácil en la organización donde se quiere implantar en relación a la estructura institucional, los planes de estudio de la institución y, por último a los contenidos y estilos pedagógicos de la organización. Escalabilidad. Capacidad de la plataforma de e-learnig de funcionar igualmente con un número pequeño o grande de usuarios. Estandarización. Posibilidad de importar y exportar cursos en formatos estándar como scorm.

La Universidad Especializada de las Américas (UDELAS), universidad estatal de la República de Panamá, al igual que las otras 24 universidades estatales y particulares, se enfrentó al impacto de la aparición repentina del COVID-19, en el mes de marzo en plena organización del inicio del I semestre del año académico 2020. Esta situación inesperada e inédita obligó a dar inicio a las clases mediante el uso de plataformas tecnológicas que podían brindar la acogida para la creación de aulas virtuales, su estructuración, implementación y conectividad con los estudiantes.

A pesar de que la UDELAS cuenta con algunas ofertas de postgrado en modalidad virtual y una plataforma, debido al repentino confinamiento obligatorio no existió el tiempo suficiente para unificar criterios tecnológicos y pedagógicos para la creación de aulas o de otras herramientas de soporte que usarían los docentes y estudiantes en el desarrollo de las clases virtuales. De lo anterior surgió la necesidad de conocer cómo se estructuraron las aulas, la organización de los contenidos, la metodología, otras herramientas empleadas, nivel de satisfacción de los involucrados y cómo se evaluaron a los estudiantes. Todas estas interrogantes y dudas fueron la base por la cual se elaboró la investigación.

La investigación servirá como parámetros en el inicio e implementación de cualquier programa de educación superior basado en la formación virtual, pues se determinaron los requerimientos, procedimientos $\mathrm{y}$ condiciones mínimas para lograr el éxito en su implementación en cualquier otra latitud con condiciones y una población similar a la que vivió la experiencia.

\section{MÉTODO}

El diseño de la presente investigación fue tipo descriptiva. En cuanto al tiempo de aplicación, de tipo transversal. La población seleccionada perteneció al universo de estudiantes de la Facultad de Educación Especial y Pedagogía de la Universidad Especializada de Las Américas, el cual fue de 4696 en el primer semestre del año 2020, y constituida específicamente por estudiantes de las Carreras de Licenciatura en Estimulación Temprana y Orientación Familiar, Licenciatura en Educación Especial, Licenciatura en Informática Educativa y Licenciatura en Docencia en Inglés.

La muestra de la investigación fue escogida bajo el método probabilístico tipo por racimo. La selección se efectuó en base al 25\% de la población de cada grupo, obteniéndose 109 estudiantes, lo que hizo muy representativa la misma.

$\mathrm{Al}$ ser una investigación tipo descriptiva, el instrumento empleado para obtener información consistió en la ENCUESTA. La misma fue estructurada en base a 14 preguntas con alternativas de repuestas: sí, no, mucho, 
poco y nada. De igual manera se adicionó preguntas complementarias para profundizar algunas respuestas. Luego de diseñada la encuesta, se envió a especialistas en el tema para la respectiva validación.

Una vez validada, la encuesta, fue insertada a las aulas de la muestra de estudiantes mediante la aplicación de Google Drive, FORMS. De tal manera, recogida la información y procesados los datos, se obtienen resultados que responden en alguna manera a las preguntas de la investigación.

\section{RESULTADOS}

La aplicación de una encuesta como herramienta del método descriptivo, permitió ofrecer datos relevantes sobre el diseño y aplicación de Aulas Virtuales en el proceso de formación universitaria en la muestra que representó la Facultad de Educación Especial y Pedagogía de UDELAS-PANAMÁ.

En este sentido, Gorduño (2006) con relación a la aplicación de las tecnologías de la Información y comunicación en la educación, manifiesta que por el impacto han producido un nuevo escenario, la educación virtual, en donde en su implementación destacan las dimensiones; de convergencia de tecnología, las relaciones entre instituciones, los contenidos de apoyo al proceso de enseñanza-aprendizaje, la recuperación de información vía internet y las necesidades del mercado de trabajo de los futuros egresados.

Siguiendo con las dimensiones señaladas anteriormente por Gorduño, sobre la convergencia tecnológica, específicamente la relación a la experiencia personal de los miembros de la muestra se pudo comprobar que la gran mayoría nunca había vivido una formación superior basada en aulas virtuales como medio de apoyo y de encuentro virtual para el logro de resultados de aprendizaje.

Así mismo, Garduño et al, señala que "lo virtual conlleva la conectividad y a la interactividad, la cual requiere la unión de distintas tecnologías de red, fenómeno al que se le ha denominado convergencia tecnológica". Con respecto al señalamiento anterior, los datos obtenidos reportaron que la mayor parte de los representantes de la muestra poseían conectividad, pero mediante el celular, herramienta cuya aplicación y sobre todo su memoria, limitaba algunas asignaciones o tareas de los módulos. Cabe resaltar que los datos indican que dicha conectividad existente no era fija en casa mediante wifi, sino por el uso del celular, como se expresó anteriormente.

El nuevo escenario producido por las tecnologías de la información y comunicación al ser novedoso como afirma Gorduño, presenta una dimensión que debemos considerar en toda formación virtual, una de ellas la convergencia tecnológica. Ante esta dimensión los datos, señalaron que para participar en forma efectiva en las clases virtuales se produjeron limitaciones y que los compañeros, vecinos o familiares, fueron los que apoyaron en la solución de algunas limitaciones y así poder resolver asignaciones en el desarrollo de las clases. Alguna información de la encuesta señala que no todas las plataformas brindan potencialidad y facilidad en su manejo y participación en clases.

Otro resultado relevante destinado a la dimensión referente a la recuperación, vía internet de la información, señalado por Gorduño, pudo visualizar poca capacitación de los docentes en el diseño, elaboración y manejo del aula virtual. En este sentido, los datos mostraron que tanto los docentes como los propios estudiantes, no poseían las mejores competencias tecnológicas para responder a las exigencias de la formación universitaria con modalidad virtual. 


\section{CONCLUSIONES}

La Universidad Especializada de las Américas al ser una institución estatal formadora de futuros profesionales a nivel nacional o internacional debe contar con una convergencia tecnológica que permita una recuperación, tratamiento y emisión de contenidos de apoyo a un proceso efectivo de enseñanza-aprendizaje tipo digital. Para ello, es necesario contar con la plataforma, segura, estable, permanente y poderosa en el desarrollo de asignaturas bajo la modalidad virtual.

Las instituciones de educación superior deben estar preparadas para enfrentar el reto que ha producido el impacto de las tecnologías de la información y comunicación sobre todo en lo referente a la existencia de normativas y procedimientos para la creación, uso y manejo de la modalidad virtual en cualquier instancia formativa $\mathrm{y}$ optar por la misma como una alternativa de opción mixta entre la presencialidad y la virtualidad en el futuro próximo.

Para que una institución cumpla con servicios de formación virtual, debe ajustarse a las exigencias de esos cambios que nos retan y para ello contar con programas de capacitación permanentes y obligatorios a estudiantes, docentes $\mathrm{y}$ administrativos de UDELAS, en el diseño, manejo e implementación de aulas virtuales como medio tecnológicos para el desarrollo y apoyo de las clases virtuales futuras. La preparación futura hacia la virtualidad exige adquirir equipos tecnológicos en todas las actividades académicas de la universidad, de tal manera que existan Bancos de docentes y estudiantes renovados que hagan frente a esos retos de la formación virtual universitaria. Por todo lo anterior se hace necesario la adquisición de equipo tecnológico e infraestructura con tecnologías que posibiliten la expedita experiencia de aprendizaje y tramitaciones administrativas con la modalidad virtual.

\section{REFERENCIAS}

Almenara, J. C., y Cejudo, M. D. C. L. (2015). Tecnologías de la Información y la Comunicación (TIC): esccabroenarios formativos y teorías del aprendizaje. Revista lasallista de investigación, 12(2), 186-193

Badia, A. y Monereo, C. (2004). La construcción de conocimiento profesional docente. Análisis de un curso de formación sobre la enseñanza estratégica. Anuario de Psicología

Belloch, C. (2012). Las Tecnologías de la Información y Comunicación en el aprendizaje. Departamento de Métodos de Investigación y Diagnóstico en Educación. Universidad de Valencia

Belloch et al. (2013). Entornos Virtuales de Aprendizaje. Recuperado de https://www.uv.es/bellochc/pdf/pwtic2. pdf_junio_2020.pdf

Cabero, J. (2006)., ¿Cuáles son las Ventajas del Aprendizaje Virtual? Recuperado de http:// sites.google.com $>$ site $>$

Cabero, J., y Barroso, J. (Coords.). (2015). Nuevos Retos en Tecnología Educativa. Madrid: Síntesis

Cabero Almenara, J., Llorente Cejudo, M. D. C., \& Vázquez Martínez, A. I. (2014). Las tipologías de MOOC: su diseño e implicaciones educativas

Díaz Barriga, F., Rigo, M.A., y Hernández, G. (2015). Experiencias de Aprendizaje. Mediadas por las Tecnologías Digitales. Pautas para Docentes y Diseñadores Educativos. México: Newton. Edición y Tecnología Educativa

Garduño Vera, R. (2006). Objetos de aprendizaje en la educación virtual: una aproximación en bibliotecología. Investigación bibliotecológica, 20(41), 161-194

Hernández, D., Ortiz, G., y Nájera, O. (2019). Futuros Digitales. Exploraciones Socioculturales de las TIC. México: Juan Pablos Editor 
Meneses, G., (2007). NTIC, Interacción y Aprendizaje en la Universidad. Las Nuevas Tecnologías de la Información.5-54. Recuperado de https://www.tesisenred.net $>\mathrm{h}$

Ochoa, X., y Cordero, S. (2002). Las Nuevas Tecnologías de la Información y la Comunicación. Recuperado de http://www.ruv.itesm.mx/especiales/cite la/documentos/material/módulos/módul os2/contendioii.htm_agosto_2020.pdf

Organización de las Naciones Unidas para la Educación, la Ciencia y la Cultura
(UNESCO) (sf), "Educación Superior Digital, disponible en http://UNESCO.org/>tema5/>edu/ed_juli o_2020.pdf

Pere, M., (2013). Impacto de las TIC en la Educación: Funciones y Limitaciones. Revista 3 Ciencias. Departamento de Pedagogía. Universidad de Barcelona. Recuperado de https:// Dialnet.unirioja.es $>$ art..._junio_2020.pdf

Thompson, A., y Strickland, A.J (2004). Administración Estratégica. Undécima edición. McGraw-Hill. México 